\title{
The End of Crypto
}

\author{
Jonathan Zittrain
}

Professor of Law School and Professor of Computer Science, Harvard University, USA

\begin{abstract}
This talk will reflect on the core purposes of cryptology, and the extent to which those purposes are served - and servable - in today's digital environment.
\end{abstract}

